\title{
Infants hospitalized for acute COVID-19: disease severity in a multicenter cohort study
}

\author{
Joanna Merckx ${ }^{1}$ (D) Shaun K. Morris ${ }^{2} \cdot$ Ari Bitnun $^{2} \cdot$ Peter Gill $^{2} \cdot$ Tala El Tal $^{2} \cdot$ Ronald M. Laxer $^{2}$. \\ Ann $\mathrm{Yeh}^{2} \cdot{\text { Carmen } \mathrm{Yea}^{2} \cdot \text { Rolando Ulloa-Gutierrez }}^{3} \cdot$ Helena Brenes-Chacon $^{3}$. Adriana Yock-Corrales ${ }^{3}$. \\ Gabriela Ivankovich-Escoto ${ }^{3}$ - Alejandra Soriano-Fallas ${ }^{3} \cdot$ Marcela Hernandez-de Mezerville $^{3} \cdot$ Jesse Papenburg ${ }^{1,4}$. \\ Marie-Astrid Lefebvre ${ }^{4}$ Alireza Nateghian ${ }^{5} \cdot$ Behzad Haghighi Aski $^{5} \cdot$ Ali Manafi $^{5} \cdot$ Rachel Dwilow $^{6}$. Jared Bullard ${ }^{6}$. \\ Suzette Cooke ${ }^{7} \cdot$ Tammie Dewan $^{7} \cdot$ Lea Restivo $^{7} \cdot$ Alison Lopez $^{8} \cdot$ Manish Sadarangani $^{8,9,10} \cdot$ Ashley Roberts $^{8,9}$. \\ Michelle Barton $^{11}$. Dara Petel ${ }^{11}$. Nicole Le Saux ${ }^{12}$. Jennifer Bowes ${ }^{12} \cdot$ Rupeena Purewal $^{13}$. Janell Lautermilch ${ }^{13}$. \\ Sarah Tehseen ${ }^{13} \cdot$ Ann Bayliss $^{14}$. Jacqueline K. Wong ${ }^{15} \cdot$ Isabelle Viel-Thériault $^{16} \cdot$ Dominique Piche $^{17}$. \\ Karina A. Top ${ }^{17}$. Kirk Leifso ${ }^{18}$. Cheryl Foo ${ }^{19}$. Luc Panetta ${ }^{20}$. Joan Robinson ${ }^{21}$
}

Received: 24 November 2021 / Revised: 7 February 2022 / Accepted: 13 February 2022 / Published online: 25 February 2022 (c) The Author(s), under exclusive licence to Springer-Verlag GmbH Germany, part of Springer Nature 2022

\begin{abstract}
Age is the most important determinant of COVID-19 severity. Infectious disease severity by age is typically J-shaped, with infants and the elderly carrying a high burden of disease. We report on the comparative disease severity between infants and older children in a multicenter retrospective cohort study of children 0 to 17 years old admitted for acute COVID-19 from February 2020 through May 2021 in 17 pediatric hospitals. We compare clinical and laboratory characteristics and estimate the association between age group and disease severity using ordinal logistic regression. We found that infants comprised one-third of cases, but were admitted for a shorter period (median 3 days IQR 2-5 versus 4 days IQR 2-7), had a lower likelihood to have an increased C-reactive protein, and had half the odds of older children of having severe or critical disease (OR 0.50 (95\% confidence interval 0.32-0.78)).

Conclusion: When compared to older children, there appeared to be a lower threshold to admit infants but their length of stay was shorter and they had lower odds than older children of progressing to severe or critical disease.

\section{What is Known:}

- A small proportion of children infected with SARS-CoV-2 require hospitalization for acute COVID-19 with a subgroup needing specialized intensive care to treat more severe disease.

- For most infectious diseases including viral respiratory tract infections, disease severity by age is J-shaped, with infants having more severe disease compared to older children.

What is New:

- One-third of admitted children for acute COVID-19 during the first 14 months of the pandemic were infants.

- Infants had half the odds of older children of having severe or critical disease.
\end{abstract}

Keywords Infants $\cdot$ COVID-19 $\cdot$ Disease severity $\cdot$ Age

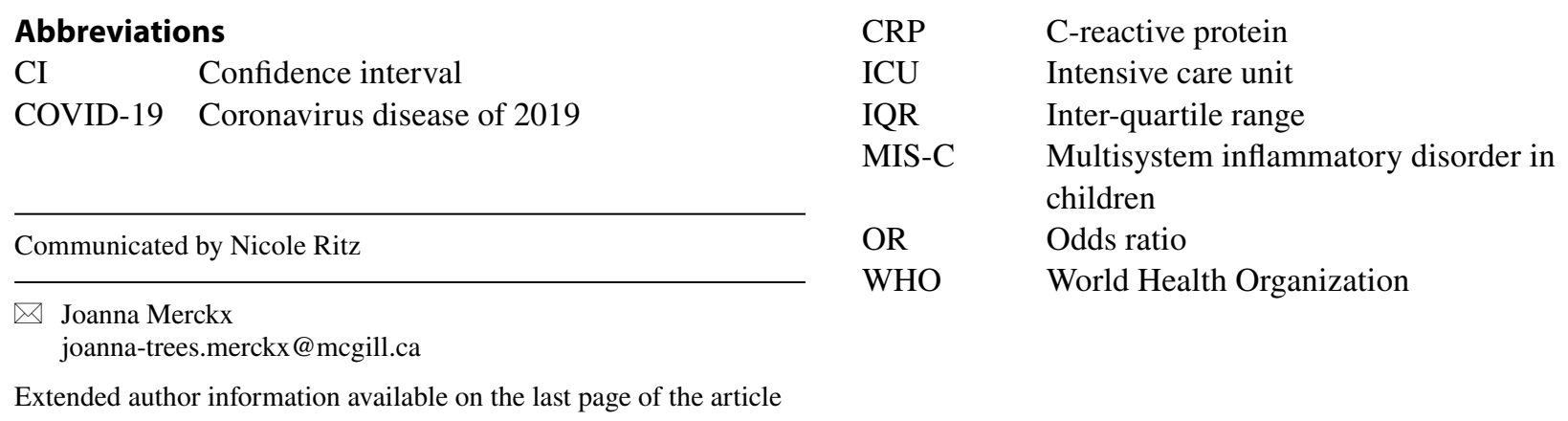




\section{Introduction}

The distribution of infectious disease severity by age is typically J-shaped [1]. Amongst children, neonates and infants have a high burden of disease [2], particularly with respiratory pathogens, and have the highest hospitalization rates [3]. The objective of this study was to determine whether this is true for coronavirus disease 2019 (COVID-19) in hospitalized children.

\section{Methods}

Seventeen pediatric hospitals (15 Canadian and one each in Iran and Costa Rica) included children up to 17 years of age, admitted February 1, 2020, through May 31, 2021, with detection of SARS-CoV-2. Patients with incidental SARSCoV-2 infection (it was not the reason for admission and did not prolong hospitalization) or who met World Health Organization (WHO) criteria for multisystem inflammatory syndrome in children (MIS-C) [4] were excluded as acute COVID-19 was not the reason for admission. Following ethics approval at all sites, data were extracted into REDCap from medical records including demographics, role of SARSCoV-2 in admission, comorbidities (prematurity, malignancy, asthma, chronic pulmonary, heart or renal disease, obesity, or significant congenital anomalies), antibiotic use, clinical presentation, and course.

Cases were defined as mild (ward admission without supplemental oxygen), severe (ward admission with supplemental oxygen), or critical (admission to ICU or death) [5].

Children were divided into infants (up to 11 months of age) versus older children for the primary analysis. For those older than 90 days, only month of birth was recorded, so age was the number of months between the birth and admission month. Sensitivity analyses assessed outcomes (i) in three age groups: up to 29 days, 30 days to 11 months, and 12 months or older and (ii) in infants 0 to 5 months versus 6 to 11 months old.

Descriptive statistics were used to summarize baseline characteristics of patients and comparative statistics was performed applying Kruskal-Wallis and chi-square test. Associations between age group and disease severity were examined using ordinal logistic regression in STATA 13 (StataCorp), estimating the odds of mild versus severe or critical disease.

\section{Results}

There were 117 (36\%) infants and 207 (64\%) older children admitted for COVID-19 (Fig. 1) after incidental SARSCoV-2 $(N=346)$ and MIS-C cases $(N=144)$ were excluded

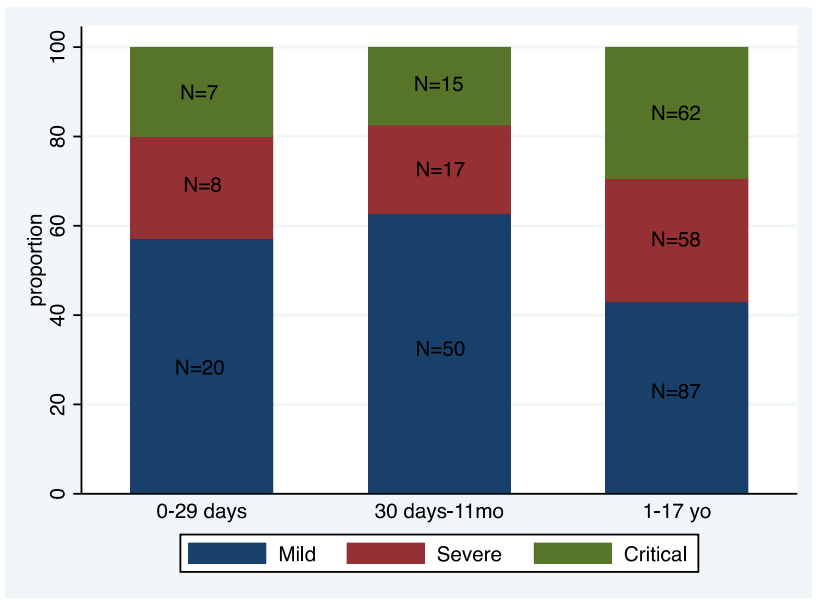

$\mathrm{N}=$ absolute number of children included in the group

Fig. 1 Proportion and absolute number of neonates, infants, and children with mild, severe, and critical outcome COVID-19 admission

(Supplement Flow Chart Fig. 1). Eighty-six infants (74\%) had no comorbidities, of which 55 (64\%) had mild, 18 (21\%) severe, and 13 (15\%) critical disease, compared to $57(28 \%)$ older children, including 32 (56\%) with mild, 13 (23\%) with severe, and 12 (21\%) having critical disease. One older child with severe disease was admitted to ICU for reasons unrelated to COVID-19.

Of $31(26 \%)$ infants with comorbidities, 15 (48\%) had mild, 7 (23\%) severe, and 9 (29\%) critical disease. This contrasted to 150 (72\%) older children with comorbidities, with mild, severe, and critical disease in 55 (37\%), 45 (30\%), and $50(33 \%)$, respectively.

Symptoms attributable to COVID-19 were similar in infants versus older children (Table 1). CRP was much more likely to be elevated in older children than in infants $(67 \%$ versus $15 \%$; $p$-value $<0.0001)$. Similar proportions received antibiotics ( $67 \%$ of infants versus $60 \%$ of older children) (Table 1). The indication was possible bacterial pneumonia in $21 \%$ of infants given antibiotics versus $36 \%$ of older children. Bacterial coinfection was confirmed in 9 infants $(8 \%)$ versus 6 older children (3\%). Proportions of confirmed viral co-infection were similar in infants and older children, $8 \%$ and $9 \%$, respectively.

Length of stay was shorter in infants (median 3 days IQR $2-5$ versus 4 days IQR $2-7)(p$-value $=0.0043)$. For infants, the odds of having severe or critical disease was half that of older children (OR 0.50 (95\%CI 0.32-0.78)). Compared to older children, the ORs for infants up to 29 days old and 30 days to 11 months were 0.56 (95\% CI 0.28-1.11) and 0.48 (95\% CI 0.29-0.79), respectively. There was insufficient evidence for differing disease severity in infants up to 5 months versus 6 to 11 months old (Supplement). Six deaths occurred in children 14 months to 9 years old; all had comorbidities. 
Table 1 Symptoms, peak CRP values, and use of antibiotics in hospitalized infants versus older children with a primary diagnosis of acute COVID-19

\begin{tabular}{lll}
\hline & $\begin{array}{l}\text { Infants } \\
N=117\end{array}$ & $\begin{array}{l}12 \text { months to } \\
\text { age } \\
\text { agears of }\end{array}$ \\
& & $N=207$ \\
\hline Cough & $51(44 \%)$ & $135(65 \%)$ \\
Shortness of breath & $49(42 \%)$ & $125(60 \%)$ \\
Rhinitis & $49(42 \%)$ & $60(29 \%)$ \\
Vomiting & $21(18 \%)$ & $51(25 \%)$ \\
Diarrhea & $21(18 \%)$ & $49(24 \%)$ \\
Wheezing & $15(13 \%)$ & $37(18 \%)$ \\
Rash & $5(4 \%)$ & $14(7 \%)$ \\
New-onset seizures & $3(3 \%)$ & $5(2 \%)$ \\
Conjunctivitis & $0(0 \%)$ & $8(4 \%)$ \\
Splenomegaly & $0(0 \%)$ & $3(1 \%)$ \\
Hepatomegaly & $0(0 \%)$ & $2(1 \%)$ \\
Fever history & & \\
$\quad$ Fever documented in hospital & $40 / 106(38 \%)$ & $90 / 182(49 \%)$ \\
Fever prior to admission only & $39 / 106(37 \%)$ & $53 / 182(29 \%)$ \\
$\quad$ No fever & $27 / 106(25 \%)$ & $39 / 182(21 \%)$ \\
Data missing & $11 / 117$ & $25 / 207$ \\
Elevated peak CRP (> 8.0 mg/L) & $14 / 94(15 \%)$ & $114 / 169(67 \%)$ \\
Antibiotics during admission & & $82(40 \%)$ \\
$\quad$ None & $39(33 \%)$ & $75(36 \%)$ \\
$\quad$ Started for possible bacterial & $16(14 \%)$ & \\
pneumonia & & \\
Started for other possible or & $61(52 \%)$ & $49(24 \%)$ \\
proven bacterial infection & $1(1 \%)$ & \\
Data missing & & \\
\hline
\end{tabular}

$C R P$ C-reactive protein

\section{Discussion}

Over one-third of children admitted with acute COVID-19 were infants, in a period with wide circulation of wildtype virus and of the variants-of-concern alpha and delta. However, the proportion of infants with severe or critical disease was lower than for older children. As far as we are aware, this is the first study to directly compare the severity of illness in hospitalized infants versus older children. Previous studies that analyzed the severity in admitted infants reported that only 4 of 34 symptomatic infants up to 90 days of age had severe or critical disease [6] and that only 1 of 14 infants was critically ill [7].

CRP was much more likely to be elevated in older children than in infants (67\% versus $15 \%)$. CRP is a sensitive marker of inflammation even in neonates $[8,9]$ so we hypothesize a lower level of measurable inflammation in infants compared to older children admitted with COVID-19, but it may reflect admission of infants with milder disease. Less inflammation might partially explain why infants accounted for only $4 \%$ of MIS-C cases in a large series [10].

Limitations are that this study is not population based and investigated children admitted primarily to tertiary care centers. The threshold is presumably lower to admit infants versus older children with a similar severity of illness, especially if they are febrile and less than 90 days old [11]. This may explain why admitted infants in our study had less severe disease than did older children. Insufficient power prevents us to provide evidence for the rare outcome of mortality and for additional and more specific age and other subgroups. Furthermore, when the outcome is common, OR's calculated cannot be interpreted as risks.

In conclusion, contrary to what is observed in most other infectious diseases [1], SARs-CoV-2 infection is not more severe in infants admitted with acute COVID-19 compared to older children.

Supplementary information The online version contains supplementary material available at https://doi.org/10.1007/s00431-022-04422-x.

Acknowledgements The study was made possible thanks to the collaboration of the Paediatric Investigators Collaborative Network on Infections in Canada (PICNIC) and the manuscript was written on behalf of PICNIC.

Authors' contributions Dr. Merckx analyzed the data and reviewed and revised the manuscript. Dr. Barton conceptualized and designed the study, collected data, and reviewed and revised the manuscript. Drs. Morris, Bitnun, Gill, El Tal, Laxer, Yeh, Yea, Ulloa-Gutierrez, Brenes-Chacon, Yock-Corrales, IvankovichEscoto, Soriano-Fallas, Hernandez-de Mezerville, Papenburg, Lefebvre, Nateghian, Aski, Manafi, Dwilow, Bullard, Cooke, Dewan, Restivo, Lopez, Sadarangani, Roberts, Petel, Le Saux, Bowes, Purewal, Lautermilch, Tehseen, Bayliss, Wong, VielThériault, Piche, Top, Leifso, Foo, and Panetta collected data and reviewed and revised the manuscript. Dr. Robinson conceptualized and designed the study, drafted the initial manuscript, and reviewed and revised the manuscript. All authors approved the final manuscript as submitted and agree to be accountable for all aspects of the work.

Availability of data and material The data are not publicly available.

Code availability The codes are available on request.

\section{Declarations}

Ethics approval This study was performed in line with the principles of the Declaration of Helsinki. Ethics approval was obtained primarily at the University of Alberta (Pro00099426) and sequentially from all participating sites.

Consent to participate Not applicable.

Consent for publication Not applicable. 
Competing interests The authors declare no interests.

\section{References}

1. Glynn JR, Moss PA (2020) Systematic analysis of infectious disease outcomes by age shows lowest severity in school-age children. Sci data 7(1):1-3

2. Paulson KR, Kamath AM, Alam T, Bienhoff K, Abady GG, Abbas J, Abbasi-Kangevari M, Abbastabar H, Abd-Allah F, Abd-Elsalam SM, Abdoli A (2021) Global, regional, and national progress towards Sustainable Development Goal 3.2 for neonatal and child health: all-cause and cause-specific mortality findings from the Global Burden of Disease Study 2019. Lancet 398(10303):870-905

3. Goto T, Tsugawa Y, Mansbach JM, Camargo CA Jr, Hasegawa K (2016) Trends in Infectious Disease Hospitalizations in US Children, 2000 to 2012. Pediatr Infect Dis J 35(6):e158-e163

4. World Health Organization (2020) Multisystem inflammatory syndrome in children and adolescents temporally related to COVID-19. https://www.who.int/publications/i/item/multisystem-inflammatory-syndrome-in-children-and-adolescents-withcovid-19 (Accessed 10 Aug 2021)

5. Kushner LE, Schroeder AR, Kim J, Mathew R (2021) For COVID or With COVID: classification of SARS-CoV-2 hospitalizations in children. Hosp Pediatr
6. Shaiba LA, Altirkawi K, Hadid A et al (2021) COVID-19 disease in infants less than 90 days: case series. Front Pediatr 9

7. DeBiasi RL, Song X, Delaney M, Bell M, Smith K, Pershad J et al (2020) Severe coronavirus disease-2019 in children and young adults in the Washington DC Metropolitan Region. J Pediatr 223:199-203

8. Lacaze-Masmonteil T, Rosychuk RJ, Robinson JL (2014) Value of a single C-reactive protein measurement at $18 \mathrm{~h}$ of age. Arch Dis Child Educ Pract Ed 99(1):F76-F79

9. Petel D, Winters N, Gore GC et al (2018) Use of C-reactive protein to tailor antibiotic use: a systematic review and meta-analysis. BMJ Open 8(12):e022133

10. Godfred-Cato S, Tsang CA, Giovanni J et al (2021) Multisystem inflammatory syndrome in infants $<12$ months of age, United States, May 2020-January 2021. Pediatr Infect Dis J 40(7):601-605

11. Pantell RH, Roberts KB, Adams WG, Dreyer BP, Kuppermann N, O'Leary ST, Okechukwu K, Woods CR (2021) Evaluation and management of well-appearing febrile infants 8 to 60 days old. Pediatrics 148(2)

Publisher's Note Springer Nature remains neutral with regard to jurisdictional claims in published maps and institutional affiliations.

\section{Authors and Affiliations}

Joanna Merckx ${ }^{1}$ ( ) Shaun K. Morris ${ }^{2} \cdot$ Ari Bitnun $^{2} \cdot$ Peter Gill $^{2} \cdot$ Tala El Tal $^{2} \cdot$ Ronald M. Laxer $^{2}$. Ann Yeh ${ }^{2} \cdot$ Carmen $\mathrm{Yea}^{2} \cdot$ Rolando Ulloa-Gutierrez ${ }^{3} \cdot$ Helena Brenes-Chacon $^{3} \cdot$ Adriana Yock-Corrales $^{3}$. Gabriela Ivankovich-Escoto ${ }^{3}$ - Alejandra Soriano-Fallas ${ }^{3}$ - Marcela Hernandez-de Mezerville ${ }^{3}$ Jesse Papenburg ${ }^{1,4}$. Marie-Astrid Lefebvre ${ }^{4}$. Alireza Nateghian ${ }^{5}$. Behzad Haghighi Aski ${ }^{5}$. Ali Manafi ${ }^{5}$. Rachel Dwilow ${ }^{6}$. Jared Bullard ${ }^{6}$. Suzette Cooke ${ }^{7}$. Tammie Dewan ${ }^{7}$. Lea Restivo ${ }^{7}$. Alison Lopez ${ }^{8}$. Manish Sadarangani ${ }^{8,9,10}$. Ashley Roberts ${ }^{8,9}$. Michelle Barton ${ }^{11}$. Dara Petel ${ }^{11}$. Nicole Le Saux ${ }^{12}$. Jennifer Bowes ${ }^{12}$. Rupeena Purewal ${ }^{13}$. Janell Lautermilch ${ }^{13}$. Sarah Tehseen ${ }^{13} \cdot$ Ann Bayliss $^{14}$. Jacqueline K. Wong ${ }^{15} \cdot$ Isabelle Viel-Thériault ${ }^{16}$. Dominique Piche ${ }^{17}$. Karina A. Top ${ }^{17} \cdot$ Kirk Leifso $^{18}$. Cheryl Foo ${ }^{19}$. Luc Panetta $^{20}$. Joan Robinson ${ }^{21}$

\author{
Shaun K. Morris \\ shaun.morris@sickkids.ca \\ Ari Bitnun \\ ari.bitnun@sickkids.ca \\ Peter Gill \\ peter.gill@sickkids.ca \\ Tala El Tal \\ tala.eltal@sickkids.ca \\ Ronald M. Laxer \\ ronald.laxer@sickkids.ca \\ Ann Yeh \\ ann.yeh@sickkids.ca \\ Carmen Yea \\ carmen.yea@sickkids.ca \\ Rolando Ulloa-Gutierrez \\ rolandoug@gmail.com
}

Helena Brenes-Chacon

helenabrenes@gmail.com

Adriana Yock-Corrales

adriyock@gmail.com

Gabriela Ivankovich-Escoto

givankovich@gmail.com

Alejandra Soriano-Fallas

alejsoriano@gmail.com

Marcela Hernandez-de Mezerville

marcelahdm@hotmail.com

Jesse Papenburg

jesse.papenburg@mail.mcgill.ca

Marie-Astrid Lefebvre

marie-astrid.lefebvre@muhc.mcgill.ca

Alireza Nateghian

alirezanateghian41@gmail.com 
Behzad Haghighi Aski

Haghighi.b@iums.ac.ir

Ali Manafi

manafi.a@iums.ac.ir

Rachel Dwilow

Rachel.Dwilow@umanitoba.ca

Jared Bullard

Jared.Bullard@gov.mb.ca

Suzette Cooke

Suzette.Cooke@albertahealthservices.ca

Tammie Dewan

tammie.dewan@albertahealthservices.ca

Lea Restivo

Lea.Restivo@albertahealthservices.ca

Alison Lopez

alison.lopez17@gmail.com

Manish Sadarangani

msadarangani@bcchr.ubc.ca

Ashley Roberts

aroberts6@cw.bc.ca

Michelle Barton

Michelle.BartonForbes@1hsc.on.ca

Dara Petel

darapetel@gmail.com

Nicole Le Saux

lesaux@cheo.on.ca

Jennifer Bowes

jbowes@cheo.on.ca

Rupeena Purewal

rupeenapurewal@gmail.com

Janell Lautermilch

jt1494@mail.usask.ca

Sarah Tehseen

Sarah.Tehseen@saskhealthauthority.ca

Ann Bayliss

Ann.Bayliss@thp.ca

Jacqueline K. Wong

wongj37@mcmaster.ca

Isabelle Viel-Thériault

isabelle.viel-theriault.1@ulaval.ca

Dominique Piche

Dominique.Piche@dal.ca

Karina A. Top

karina.top@dal.ca

Kirk Leifso

Kirk.Leifso@kingstonhsc.ca

Cheryl Foo

cpz.foo@gmail.com
Luc Panetta

luc.panetta@hotmail.fr

Joan Robinson

jr3@ualberta.ca

1 Department of Epidemiology, Biostatistics and Occupational Health, McGill University, McGill College, Suite 1200, Montreal, QC H3A 1G1, Canada

2 Department of Pediatrics, University of Toronto, Toronto, ON, Canada

3 Department of Pediatrics, Caja Costarricense de Seguro Social (CCSS), Hospital Nacional de Niños Dr. Carlos Sáenz Herrera, San José, Costa Rica

4 Department of Pediatrics, McGill University, Montreal, QC, Canada

5 Department of Pediatrics, University of Medical Sciences, Tehran, Iran

6 Department of Pediatrics, University of Manitoba, Winnipeg, MB, Canada

7 Department of Pediatrics, University of Calgary, Calgary, AB, Canada

8 British Columbia Children's Hospital, Vancouver, BC, Canada

9 Department of Pediatrics, University of British Columbia, Vancouver, BC, Canada

10 Vaccine Evaluation Center, BC Children's Hospital Research Institute, Vancouver, BC, Canada

11 Department of Pediatrics, Western University, London, ON, Canada

12 Department of Pediatrics, University of Ottawa, Ottawa, ON, Canada

13 Department of Pediatrics, University of Saskatchewan, Saskatoon, SK, Canada

14 Department of Pediatrics, Trillium Health Partners, Mississauga, ON, Canada

15 Department of Pediatrics, McMaster University, Hamilton, ON, Canada

16 Department of Pediatrics, CHU de Québec-Université Laval, Québec, QC, Canada

17 Department of Pediatrics, Dalhousie University, Halifax, NS, Canada

18 Department of Pediatrics, Queen's University, Kingston, ON, Canada

19 Department of Pediatrics, Memorial University, St John's, Newfoundland and Labrador, Canada

20 Department of Pediatrics, Université de Montréal, Montreal, QC, Canada

21 Department of Pediatrics, University of Alberta, Edmonton, AB, Canada 